\title{
Higgs boson production in photon-photon interactions with proton, light-ion, and heavy-ion beams at current and future colliders
}

\author{
David d'Enterria* \\ CERN, European Organization for Nuclear Research, 1211 Geneva, Switzerland \\ Daniel E. Martins ${ }^{\dagger}$ \\ UFRJ, Univ. Federal do Rio de Janeiro, 21941-901 Rio de Janeiro, RJ, Brazil \\ Patricia Rebello Teles \\ UERJ, Univ. do Estado do Rio de Janeiro, 20550-900 Rio de Janeiro, RJ, Brazil
}

(Received 3 May 2019; accepted 28 January 2020; published 24 February 2020)

\begin{abstract}
The production of the Higgs boson in photon-photon interactions with proton and nucleus beams at three planned or proposed future CERN colliders - the high-luminosity Large Hadron Collider (HL-LHC), the high-energy LHC (HE-LHC), and the Future Circular Collider (FCC) - is studied. The cross sections for the process $\mathrm{AA} \stackrel{\gamma \gamma}{\rightarrow}(\mathrm{A}) \mathrm{H}(\mathrm{A})$, with the ions $\mathrm{A}$ surviving the interaction and the Higgs scalar exclusively produced, are computed with MADGRAPH 5 modified to include the corresponding elastic $\gamma$ fluxes, for $\mathrm{Pb}-\mathrm{Pb}, \mathrm{Xe}-\mathrm{Xe}, \mathrm{Kr}-\mathrm{Kr}$, Ar-Ar, O-O, p- $\mathrm{Pb}$, and p-p over the nucleon-nucleon collision energy range $\sqrt{s_{\mathrm{NN}}} \approx 3-100 \mathrm{TeV}$. Simulations of the $\gamma \gamma \rightarrow \mathrm{H} \rightarrow \mathrm{b} \overline{\mathrm{b}}$ decay mode-including realistic (mis)tagging and reconstruction efficiencies for the final-state b-jets, as well as appropriate kinematical selection criteria to reduce the similarly computed $\gamma \gamma \rightarrow \mathrm{b} \bar{b}, \mathrm{c} \overline{\mathrm{c}}, \mathrm{q} \overline{\mathrm{q}}$ continuum backgrounds—have been carried out. Taking into account the expected luminosities for all systems, the yields, and significances for observing the Higgs boson in ultraperipheral collisions (UPCs) are estimated. At the HL-LHC and HE-LHC, the colliding systems with larger Higgs significance are $\mathrm{Ar}-\mathrm{Ar}(6.3 \mathrm{TeV})$ and $\mathrm{Kr}-\mathrm{Kr}$ (12.5 TeV), respectively, but $3 \sigma$ evidence for two-photon Higgs production would require 200 and 30 times larger integrated luminosities than those planned today at both machines. Factors of 10 can be gained by running for a year, rather than the typical 1-month heavy-ion LHC operation, but the process will likely remain unobserved until a higher energy hadron collider, such as the FCC, is built. In the latter machine, the $5 \sigma$ observation of Higgs production in UPCs is feasible in just the first nominal run of $\mathrm{Pb}-\mathrm{Pb}$ and $\mathrm{p}-\mathrm{Pb}$ collisions at $\sqrt{s_{\mathrm{NN}}}=39$ and $63 \mathrm{TeV}$, respectively.
\end{abstract}

DOI: 10.1103/PhysRevD.101.033009

\section{INTRODUCTION}

Heavy ions accelerated at high energies are surrounded by huge electromagnetic (e.m.) fields generated by the collective action of their $Z$ individual proton charges. In the equivalent photon approximation (EPA) [1], such strong e.m. fields can be identified as quasireal photon beams with very low virtualities $Q^{2}<1 / R_{A}^{2}$ and large longitudinal energies of up to $\omega_{\max } \approx \gamma_{L} / R_{A}$, where $R_{A}$ is the radius of

\footnotetext{
*david.d'enterria@cern.ch †dan.ernani@gmail.com

"patricia.rebello.teles@cern.ch
}

Published by the American Physical Society under the terms of the Creative Commons Attribution 4.0 International license. Further distribution of this work must maintain attribution to the author(s) and the published article's title, journal citation, and DOI. Funded by SCOAP ${ }^{3}$. the charge and $\gamma_{L}=E_{\text {beam }} / m_{N, p}$ is the beam Lorentz factor for nucleon or proton mass $m_{N, p}=0.9315,0.9382 \mathrm{GeV}$ $[2,3]$. On the one hand, since the photon flux scales as the squared charge of each colliding particle, photon-photon cross sections are enhanced millions of times for heavy ions (up to $Z^{4} \approx 5 \times 10^{7}$ for $\mathrm{Pb}-\mathrm{Pb}$ ) compared to proton or electron beams. On the other hand, proton (and lighter ions) features larger $\omega_{\max }$ values thanks to their lower radii $R_{A}$ and larger beam $\gamma_{L}$ factors and can thereby reach higher photon-photon center-of-mass (c.m.) energies. At the energies of the Large Hadron Collider (LHC), photons emitted from nuclei (with radii $R_{A} \approx 1.2 A^{1 / 3} \mathrm{fm}$ ) are almost on shell (virtuality $Q<0.06 \mathrm{GeV}$, for mass numbers $A>16)$ and reach longitudinal energies of up to hundreds of $\mathrm{GeV}$, whereas photon fluxes from protons $\left(R_{A} \approx 0.7 \mathrm{fm}\right)$ have larger virtualities, $Q \approx 0.28 \mathrm{GeV}$, and longitudinal energies in the $\mathrm{TeV}$ range [3]. Table I summarizes the relevant characteristics of photon-photon 
TABLE I. Summary of the characteristics of photon-photon collisions in ultraperipheral proton and nuclear collisions at the HL-LHC, HE-LHC, and FCC [4-6]. (i) Nucleon-nucleon c.m. energy $\sqrt{s_{\mathrm{NN}}}$, (ii) integrated luminosity per run $\mathcal{L}_{\text {int }}$, (iii) beam energies $E_{\text {beam }}$, (iv) Lorentz factor $\gamma_{L}$, (v) effective charge radius $R_{A}$, (vi) photon "maximum" energy $\omega_{\max }$ in the c.m. frame, and (vii) "maximum" photon-photon c.m. energy $\sqrt{s_{\gamma \gamma}^{\max }}$. The last two columns list the $\gamma \gamma \rightarrow \mathrm{H}$ cross sections and the expected number of Higgs events for the quoted $\mathcal{L}_{\text {int }}$ per system.

\begin{tabular}{|c|c|c|c|c|c|c|c|c|c|}
\hline System & $\sqrt{s_{\mathrm{NN}}}$ & $\mathcal{L}_{\text {int }}$ & $E_{\text {beam } 1}+E_{\text {beam } 2}$ & $\gamma_{L}$ & $R_{A}$ & $\omega_{\max }$ & $\sqrt{s_{\gamma \gamma}^{\max }}$ & $\sigma(\gamma \gamma \rightarrow \mathrm{H})$ & $\mathrm{N}(\gamma \gamma \rightarrow \mathrm{H})$ \\
\hline $\mathrm{b}-\mathrm{Pb}$ & $5.5 \mathrm{TeV}$ & $10 \mathrm{nb}^{-1}$ & $2.75+2.75 \mathrm{TeV}$ & 2950 & $7.1 \mathrm{fm}$ & $80 \mathrm{GeV}$ & $160 \mathrm{GeV}$ & $15 \mathrm{pb}$ & 0.15 \\
\hline $\mathrm{Xe}-\mathrm{Xe}$ & $5.86 \mathrm{TeV}$ & $30 \mathrm{nb}^{-1}$ & $2.93+2.93 \mathrm{TeV}$ & 3150 & $6.1 \mathrm{fm}$ & $100 \mathrm{GeV}$ & $200 \mathrm{GeV}$ & $7 \mathrm{pb}$ & 0.21 \\
\hline $\mathrm{Kr}-\mathrm{Kr}$ & $6.46 \mathrm{TeV}$ & $120 \mathrm{nb}^{-1}$ & $3.23+3.23 \mathrm{TeV}$ & 3470 & $5.1 \mathrm{fm}$ & $136 \mathrm{GeV}$ & $272 \mathrm{GeV}$ & $3 \mathrm{pb}$ & 0.36 \\
\hline $\mathrm{Ar}-\mathrm{Ar}$ & $6.3 \mathrm{TeV}$ & $1.1 \mathrm{pb}^{-1}$ & $3.15+3.15 \mathrm{TeV}$ & 3400 & $4.1 \mathrm{fm}$ & $165 \mathrm{GeV}$ & $330 \mathrm{GeV}$ & $0.36 \mathrm{pb}$ & 0.40 \\
\hline $\mathrm{O}-\mathrm{O}$ & $7.0 \mathrm{TeV}$ & $3.0 \mathrm{pb}^{-1}$ & $3.5+3.5 \mathrm{TeV}$ & 3750 & $3.1 \mathrm{fm}$ & $240 \mathrm{GeV}$ & $490 \mathrm{GeV}$ & $35 \mathrm{fb}$ & 0.11 \\
\hline $\mathrm{p}-\mathrm{Pb}$ & 8.8 TeV & $1 \mathrm{pb}^{-1}$ & $7.0+2.75 \mathrm{TeV}$ & 7450,2950 & $0.7,7.1 \mathrm{fm}$ & $2.45 \mathrm{TeV}, 80 \mathrm{GeV}$ & $2.6 \mathrm{TeV}$ & $0.17 \mathrm{pb}$ & 0.17 \\
\hline p-p & $14 \mathrm{TeV}$ & $1 \mathrm{fb}^{-1}$ & $7.0+7.0 \mathrm{TeV}$ & 7450 & $0.7 \mathrm{fm}$ & $2.45 \mathrm{TeV}$ & $4.5 \mathrm{TeV}$ & $0.18 \mathrm{fb}$ & 0.18 \\
\hline $\mathrm{b}-\mathrm{Pb}$ & $10.6 \mathrm{TeV}$ & $10 \mathrm{nb}^{-1}$ & $5.3+5.3 \mathrm{TeV}$ & 5700 & $7.1 \mathrm{fm}$ & $160 \mathrm{GeV}$ & $320 \mathrm{GeV}$ & $150 \mathrm{pb}$ & 1.5 \\
\hline $\mathrm{Xe}-\mathrm{Xe}$ & $11.5 \mathrm{TeV}$ & $30 \mathrm{nb}^{-1}$ & $5.75+5.75 \mathrm{TeV}$ & 6200 & $6.1 \mathrm{fm}$ & $200 \mathrm{GeV}$ & $400 \mathrm{GeV}$ & $60 \mathrm{pb}$ & 1.8 \\
\hline $\mathrm{Kr}-\mathrm{Kr}$ & $12.5 \mathrm{TeV}$ & $120 \mathrm{nb}^{-1}$ & $6.25+6.25 \mathrm{TeV}$ & 6700 & $5.1 \mathrm{fm}$ & $260 \mathrm{GeV}$ & $530 \mathrm{GeV}$ & $20 \mathrm{pb}$ & 2.4 \\
\hline $\mathrm{Ar}-\mathrm{Ar}$ & $12.1 \mathrm{TeV}$ & $1.1 \mathrm{pb}^{-1}$ & $6.05+6.05 \mathrm{TeV}$ & 6500 & $4.1 \mathrm{fm}$ & $320 \mathrm{GeV}$ & $640 \mathrm{GeV}$ & $1.7 \mathrm{pb}$ & 1.9 \\
\hline $\mathrm{O}-\mathrm{O}$ & $13.5 \mathrm{TeV}$ & $3.0 \mathrm{pb}^{-1}$ & $6.75+6.75 \mathrm{TeV}$ & 7300 & $3.1 \mathrm{fm}$ & $470 \mathrm{GeV}$ & $940 \mathrm{GeV}$ & $0.11 \mathrm{pb}$ & 0.33 \\
\hline $\mathrm{p}-\mathrm{Pb}$ & $18.8 \mathrm{TeV}$ & $1 \mathrm{pb}^{-1}$ & $13.5+5.3 \mathrm{TeV}$ & 14400,5700 & $0.7,7.1 \mathrm{fm}$ & $4.1 \mathrm{TeV}, 160 \mathrm{GeV}$ & $4.2 \mathrm{TeV}$ & $0.45 \mathrm{pb}$ & 0.45 \\
\hline p-p & $27 \mathrm{TeV}$ & $1 \mathrm{fb}^{-1}$ & $13.5+13.5 \mathrm{TeV}$ & 14400 & $0.7 \mathrm{fm}$ & $4.1 \mathrm{TeV}$ & $8.2 \mathrm{TeV}$ & $0.30 \mathrm{fb}$ & 0.30 \\
\hline $\mathrm{Pb}-\mathrm{Pb}$ & $39 \mathrm{TeV}$ & $110 \mathrm{nb}^{-1}$ & $19.5+19.5 \mathrm{TeV}$ & 21000 & $7.1 \mathrm{fm}$ & $600 \mathrm{GeV}$ & $1.2 \mathrm{TeV}$ & $1.8 \mathrm{nb}$ & 200 \\
\hline $\mathrm{p}-\mathrm{Pb}$ & $63 \mathrm{TeV}$ & $29 \mathrm{pb}^{-1}$ & $50 .+19.5 \mathrm{TeV}$ & 53300,21000 & $0.7,7.1 \mathrm{fm}$ & $15.2 \mathrm{TeV}, 600 \mathrm{GeV}$ & $15.8 \mathrm{TeV}$ & $1.5 \mathrm{pb}$ & 45 \\
\hline p-p & $100 \mathrm{TeV}$ & $1 \mathrm{fb}^{-1}$ & $50 .+50 . \mathrm{TeV}$ & 53300 & $0.7 \mathrm{fm}$ & $15.2 \mathrm{TeV}$ & $30.5 \mathrm{TeV}$ & $0.70 \mathrm{fb}$ & 0.70 \\
\hline
\end{tabular}

collisions in ultraperipheral collisions (UPCs) of proton and nuclear beams at three planned or proposed CERN future hadron colliders: the high-luminosity LHC (HL-LHC) [4], the high-energy LHC (HE-LHC) [4,5], and the Future Circular Collider (FCC) [6]. The beam luminosities for light and heavy ions considered here are those discussed in Refs. $[4,6]$. Although the beam luminosities for p-p are 7 orders of magnitude larger than those for $\mathrm{Pb}-\mathrm{Pb}$, the running conditions with multiple pileup p-p collisions per bunch crossing hinder the measurement of exclusive $\gamma \gamma$ interactions with central masses at $125 \mathrm{GeV}$ (unless one installs, in the LHC case, very forward proton taggers at $420 \mathrm{~m}$ inside the tunnel, ${ }^{1}$ with 10 -picosecond time resolution [7]). Thus, in the present study, we take $\mathcal{L}_{\text {int }}=1 \mathrm{fb}^{-1}$ as the value potentially integrated under low-pileup conditions that allow the reconstruction of exclusive photonphoton final states in p-p collisions. In all cases in Table I, one can see that the maximum photon-photon c.m. energy reaches above the kinematical threshold for Higgs boson production, $\sqrt{s_{\gamma \gamma}^{\max }} \gtrsim m_{\mathrm{H}}=125 \mathrm{GeV}$, through the process depicted in Fig. 1 (left). The observation of the $\gamma \gamma \rightarrow \mathrm{H}$ process would provide, first, an independent measurement of the H-photon loop-induced coupling based not on the Higgs decay (as measured at the LHC [8]) but on its

\footnotetext{
${ }^{1}$ We note that a similar forward tagging of lead ions at the LHC is impossible, given that the ions carry a much larger longitudinal momentum, $p_{L}=5.5 \mathrm{TeV} \times \mathrm{A} \approx 600 \mathrm{TeV}$, than the protons, and thereby are barely deflected after a photonphoton interaction.
}

$s$-channel production mode. In addition, precise measurements of the $\Gamma(\mathrm{H} \rightarrow \gamma \gamma)$ partial width derived from $\sigma(\gamma \gamma \rightarrow \mathrm{H} \rightarrow \mathrm{b} \bar{b}) \propto \Gamma(\mathrm{H} \rightarrow \gamma \gamma) \cdot \mathrm{BR}(\mathrm{H} \rightarrow \mathrm{b} \bar{b})$, and of the Higgs branching ratio $\mathrm{BR}(\mathrm{H} \rightarrow \gamma \gamma)$ determined at a future $e^{+} e^{-}$collider, would also provide a model-independent extraction of the total Higgs width, via $\Gamma_{\mathrm{H}}^{\mathrm{tot}}=\Gamma(\mathrm{H} \rightarrow \gamma \gamma) /$ $\mathrm{BR}(\mathrm{H} \rightarrow \gamma \gamma)[9]$.

The possibility to produce the Higgs boson by exploiting the huge photon fields in UPCs of ions, $\mathrm{AA} \stackrel{\gamma \gamma}{\rightarrow}(\mathrm{A}) \mathrm{H}(\mathrm{A})$, where the scalar boson is produced at midrapidity and the colliding ions (A) survive their electromagnetic interaction (Fig. 1, left), was first considered 30 years ago in several works [10]. Detailed studies of the actual measurement of UPC production of the Higgs boson in its dominant $b \bar{b}$ decay mode, including realistic experimental acceptance and efficiencies for the signal and the $\gamma \gamma \rightarrow b \bar{b}, c \bar{c}, q \bar{q}$ continuum backgrounds (Fig. 1, right), were first presented in Ref. [11] for ultraperipheral proton-nucleus (p-A) and

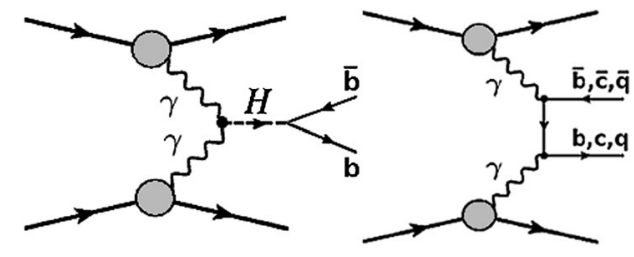

FIG. 1. Diagrams for the exclusive two-photon production of the Higgs boson (followed by its b $\bar{b}$ decay, left), and of b-, c-, light-quark pairs (processes that share the same final state as the Higgs case, right) in ultraperipheral proton/nuclear collisions. 
nucleus-nucleus (A-A) collisions at LHC energies. This work showed that, for the nominal integrated luminosities, the scalar boson was unobservable in UPCs at the LHC unless one integrated at least 300 times more luminosity than that expected for the standard 1-month heavy-ion operation. On the other hand, similar studies [12] carried out within the FCC project have indicated that the observation of Higgs production in UPCs was clearly possible in just the first nominal run of $\mathrm{Pb}-\mathrm{Pb}$ and $\mathrm{p}-\mathrm{Pb}$ collisions at $\sqrt{s_{\mathrm{NN}}}=39$ and $63 \mathrm{TeV}$, respectively. We note also that detailed studies of $\gamma \gamma \rightarrow \mathrm{H}$ were performed in the past in the context of the photon collider project $[9,13,14]$, exploiting the polarized and monochromatic $\gamma$ beams resulting from Comptonbackscattering of laser light at future $e^{+} e^{-}$linear colliders, and with reduced backgrounds (thanks to the $\gamma$ polarization) compared to those considered in the present study. In this work here, we collect our UPC Higgs results carried out in the context of the FCC studies and discuss for the first time the conditions needed for an UPC Higgs boson measurement in the upcoming HL-LHC phase, as well as at the proposed HE-LHC with twice larger c.m. energies. Our new work includes not only higher luminosities than originally planned for the LHC, but also collisions of lighter ions (Xe-Xe, $\mathrm{Kr}-\mathrm{Kr}$, Ar-Ar, O-O) never considered before.

\section{THEORETICAL SETUP}

The MADGRAPH 5 (v.2.6.5) Monte Carlo (MC) event generator [15] is employed to compute the UPC Higgs boson and diquark continuum cross sections, modified following the implementation discussed in detail in [11], as well as to generate the corresponding events for subsequent analysis. The Higgs cross section is obtained from the convolution of the Weizsäcker-Williams EPA photon fluxes for the proton and/or ions, and the elementary $\gamma \gamma \rightarrow \mathrm{H}$ cross section (with $\mathrm{H}-\gamma$ coupling parametrized in the Higgs effective field theory [16]) via

$$
\sigma_{\mathrm{A}_{1} \mathrm{~A}_{2} \rightarrow \mathrm{H}}=\int d x_{1} d x_{2} f_{\gamma / A_{1}}\left(x_{1}\right) f_{\gamma / A_{2}}\left(x_{2}\right) \hat{\sigma}_{\gamma \gamma \rightarrow \mathrm{H}},
$$

where $x=\omega / E$ is the fraction of the energy of the incoming ion carried by each photon. The same expression is used for the $b \bar{b}, c \bar{c}, q \bar{q}$ continuums, where now the elementary $\hat{\sigma}_{\gamma \gamma \rightarrow \mathrm{b} \overline{\mathrm{b}}, \mathrm{c}, \mathrm{c}, \mathrm{q} \overline{\mathrm{q}}}$ cross sections at diquark invariant masses around the Higgs mass are directly calculated at leading order (LO) by MADGRAPH 5. For protons, the MADGRAPH 5 default $\gamma$ flux is used, given by the energy spectrum of Ref. [17],

$f_{\gamma / p}(x)=\frac{\alpha}{\pi} \frac{1-x+1 / 2 x^{2}}{x} \int_{Q_{\min }^{2}}^{\infty} \frac{Q^{2}-Q_{\min }^{2}}{Q^{4}}\left|F\left(Q^{2}\right)\right|^{2} d Q^{2}$,

where $\alpha=1 / 137, F\left(Q^{2}\right)$ is the proton e.m. form factor, and the minimum momentum transfer $Q_{\min }$ is a function of $x$ and the proton mass $m_{p}, Q_{\min }^{2} \approx\left(x m_{p}\right)^{2} /(1-x)$. For ions of charge $Z$, the photon energy spectrum, integrated over impact parameter $b$ from $b_{\min }=R_{A}$ to infinity, is [18]

$f_{\gamma / A}(x)=\frac{\alpha Z^{2}}{\pi} \frac{1}{x}\left[2 x_{i} K_{0}\left(x_{i}\right) K_{1}\left(x_{i}\right)-x_{i}^{2}\left(K_{1}^{2}\left(x_{i}\right)-K_{0}^{2}\left(x_{i}\right)\right)\right]$,

where $x_{i}=x m_{N} b_{\min }, K_{0}, K_{1}$ are the zero- and first-order modified Bessel functions of the second kind, and for the different nuclear radii $R_{A}$, we use the data from elastic lepton-nucleus collisions [19]. We exclude nuclear overlap by imposing $b_{1}>R_{A_{1}}$ and $b_{2}>R_{A_{2}}$ for each photon flux and applying a correcting factor on the final cross section that depends on the ratio of Higgs mass over $\sqrt{s_{\mathrm{NN}}}$ [20].

After cross section determination, the event generation is carried out for the dominant Higgs decay mode, $\mathrm{H} \rightarrow \mathrm{b} \overline{\mathrm{b}}$ with $56 \%$ branching fraction [21], as it is the final state that provides the largest number of signal events. The same setup is used to generate the exclusive two-photon production of b $\bar{b}$ and (misidentified) $c \bar{c}$ and light-quark ( $q \bar{q})$ jet pairs, which constitute the most important physical background for the $\mathrm{H} \rightarrow \mathrm{b} \bar{b}$ measurement. For the HL-LHC and HE-LHC systems, the analysis is carried out at the parton level only, whereas for FCC energies, we have further used PYTHIA8.2 [22] to shower and hadronize the two final-state b-jets generated, which are then reconstructed with the Durham $k_{t}$ algorithm [23] (exclusive two-jets final state) using FASTJET3.0 [24]. Given that the final state consists just of two quarks (jets) exclusively produced, without any background that can potentially bias the four-momentum jet (quark) reconstruction, and that we take into account realistic jet resolution effects in the final dijet invariant mass analysis by appropriately smearing the parton-level results, no apparent differences exist between the partonand hadron-level results, as found previously in similar FCC-ee studies $[25,26]$ where, for the same set of kinematical cuts, both results are fully consistent within statistical uncertainties.

\section{TOTAL HIGGS CROSS SECTIONS}

The computed ultraperipheral Higgs boson cross sections as a function of $\sqrt{s_{\mathrm{NN}}}$ are shown in Fig. 2 (left) and listed in the before-last column of Table I for all p-p, p-A, and A-A systems considered. All theoretical cross sections have a conservative $20 \%$ uncertainty (not quoted) to cover different charge form factors and nuclear overlap conditions $[27,28]$. We note that the quoted cross sections are purely "elastic," i.e., both incoming ions survive the e.m. interaction. As discussed in [11], $\gamma \gamma$ interactions can also be "semielastic," and/or "resolved," with one (or both) quasireal photons being radiated from individual proton(s), and/ or from individual quarks, inside the colliding ions. In this latter case, one (or both) ions breakup at very forward 

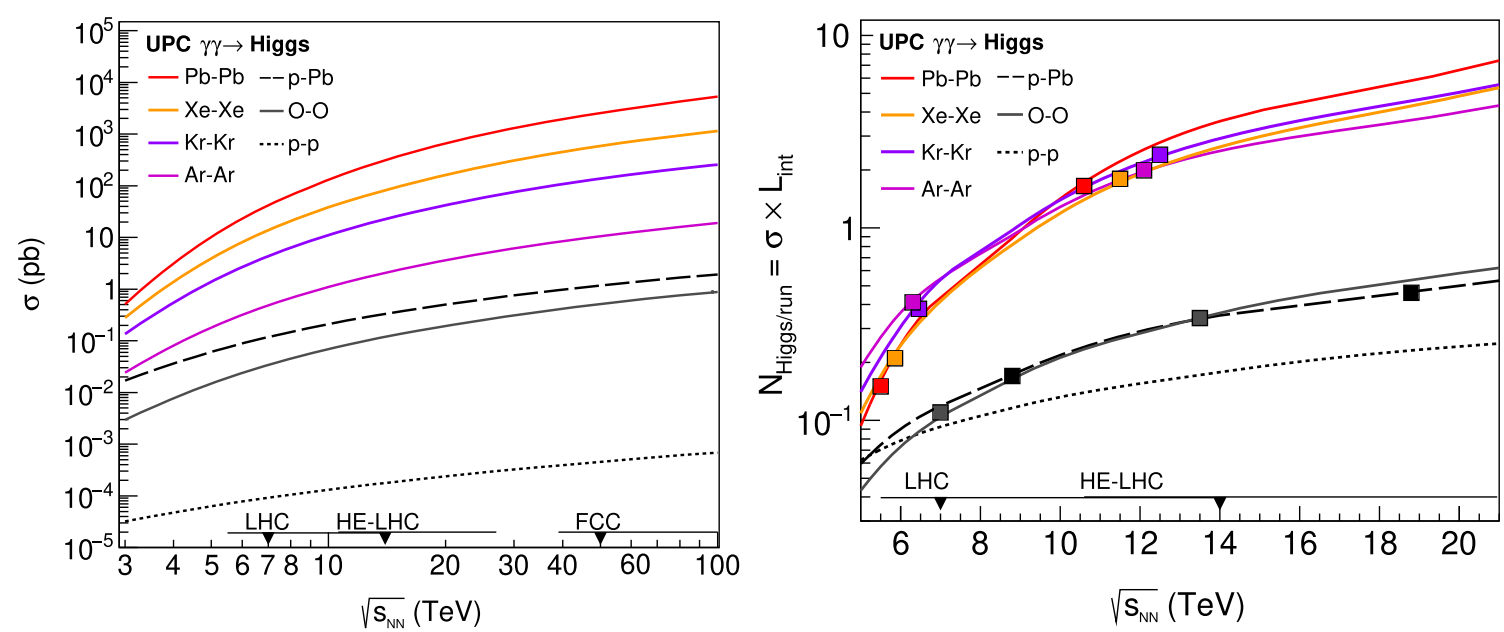

FIG. 2. Left: two-photon fusion Higgs boson cross section as a function of nucleon-nucleon c.m. energy in nuclear and proton collisions over $\sqrt{s_{\mathrm{NN}}}=3-100 \mathrm{TeV}$. Right: number of Higgs bosons produced per run (according to the integrated luminosities $\mathcal{L}_{\text {int }}$ listed in Table I) in UPCs of various colliding systems in the HL-LHC and HE-LHC energy range. The square symbols indicate the nominal $\sqrt{s_{\mathrm{NN}}}$ for each colliding system.

rapidities after photon emission, $\mathrm{AA} \stackrel{\gamma \gamma}{\rightarrow} \mathrm{AHX}$, and the Higgs boson cross sections can be enhanced by about a factor of 2 compared to the pure elastic results. We do not consider these cases here and focus on the elastic processes alone.

Figure 2 (left) indicates that, as expected, the bigger the charge of the colliding ions, the larger the UPC Higgs cross sections, but such an advantage is mitigated in terms of final yields by the correspondingly reduced beam luminosities for heavier ions. Figure 2 (right) shows the product of UPC Higgs cross section times the integrated luminosities for each colliding system in the HL-LHC and HE-LHC energy range. At the LHC, one can see that despite the fact that $\mathrm{Pb}-\mathrm{Pb}$ features the largest Higgs cross section, $\sigma(\gamma \gamma \rightarrow \mathrm{H})=15 \mathrm{pb}$, there are about two to three times more scalar bosons produced per month in Ar-Ar and $\mathrm{Kr}-\mathrm{Kr}$ collisions (0.40 vs 0.15 , last column of Table I), thanks to the comparatively larger luminosities and c.m. energies of the latter with respect to lead beams. At the HE-LHC, the Higgs cross sections are about a factor of 10 larger than at the LHC, and most colliding systems feature 1.5-2.5 Higgs bosons produced per month. The most competitive systems to try a measurement of UPC Higgs production are $\mathrm{Ar}-\mathrm{Ar}$ at $\mathrm{HL}-\mathrm{LHC}$ and $\mathrm{Kr}-\mathrm{Kr}$ at HELHC, respectively. At the FCC, the cross sections are 2 orders of magnitude larger than at the LHC, reaching $\sigma(\gamma \gamma \rightarrow \mathrm{H})=$ $1.75 \mathrm{nb}$ and $1.5 \mathrm{pb}$ in $\mathrm{Pb}-\mathrm{Pb}$ and $\mathrm{p}-\mathrm{Pb}$ collisions at $\sqrt{s_{\mathrm{NN}}}=$ 39 and $63 \mathrm{TeV}$ which, for the nominal $\mathcal{L}_{\text {int }}=110 \mathrm{nb}^{-1}$ and $29 \mathrm{pb}^{-1}$ per-month integrated luminosities, yield $\sim 200$ and 45 Higgs bosons (corresponding to 110 and 25 bosons in the $\mathrm{b} \overline{\mathrm{b}}$ decay mode), respectively.

\section{DATA ANALYSIS AND HIGGS BOSON SIGNIFICANCES}

The observation of the Higgs boson in UPCs relies on the measurement of two exclusive b-jets with invariant masses peaked at $m_{\mathrm{H}}$, on top of a background of exclusive $\gamma \gamma \rightarrow$ $\mathrm{b} \overline{\mathrm{b}}, \mathrm{c} \overline{\mathrm{c}}, \mathrm{q} \overline{\mathrm{q}}$ continuum pairs, where charm and light $(\mathrm{q}=\mathrm{u}, \mathrm{d}, \mathrm{s})$ quarks are misidentified as b-quarks. For all colliding systems, the pure MC-level background continuum cross sections over $m_{\mathrm{H}} \approx 100-150 \mathrm{GeV}$, computed with the same MADGRAPH 5 setup described above, are about 25, 200, and 500 times larger, respectively, than the Higgs signal. Experimentally, triggering the online selection of such type of events is straightforward given their unique signature characterized by two back-to-back high transverse momentum $\left(p_{T}\right)$ jets in an otherwise empty detector. The data analysis follows the strategy first proposed in Ref. [11], where more details, not repeated here, can be found. The following acceptance and reconstruction performances have been assumed: jet reconstruction over $|\eta|<2$ ( $<5$ for FCC), 7\% b-jet energy resolution (resulting in a dijet mass resolution of $\sigma_{j j} \approx 6 \mathrm{GeV}$ at the Higgs peak), $70 \%$ b-jet tagging efficiency, and 5\% (1.5\%) b-jet mistagging probability for a c (light-flavor q) quark. We note that the b-jet reconstruction and identification performances are expected to be better in the very "clean" exclusive environment of UPCs than in the current high-pileup p-p collisions at the LHC [29]. The pseudorapidity acceptance cut $|\eta|<2$ used for HL-LHC and HE-LHC keeps a large fraction of signal jets (around $80 \%$ ), while removing $2 / 3$ of the background jets, which are much more forward/backward-peaked. In terms of jet (mis)tagging efficiencies, for the double b-jet final-state of interest, these lead to a 50\% efficiency for the MCgenerated signal $(\mathcal{S})$ and a total reduction of the misidentified $c \bar{c}$ and $\mathrm{q} \overline{\mathrm{q}}$ continuum backgrounds $(\mathcal{B})$ by factors of $\sim 400$ and $\sim 4500$, respectively. The sum of remaining continuum backgrounds can be further reduced through proper kinematical cuts by requiring [11] 
(i) both jets to have transverse momenta around half the Higgs mass, $p_{T} \approx m_{\mathrm{H}} / 2 \approx 55-67 \mathrm{GeV}$, as expected for two back-to-back jets from the decay of an UPC Higgs produced almost at rest, thereby suppressing more than $95 \%$ of the continuum, while removing about half of the signal;

(ii) the angle of the jets in the helicity frame to be $|\cos \theta|<0.5$ - to exploit the fact that the angular distribution in the helicity frame of the Higgs decay b-jets is isotropic while the continuum (with quarks propagating in the $t$ - or $u$-channels) is peaked in the forward-backward directions-further suppressing the backgrounds while leaving almost untouched the number of signal events; and

(iii) the pair jet mass to be within $\pm 1.4 \sigma_{j j}$ around the Higgs mass (i.e., $116 \lesssim m_{\mathrm{b} \bar{b}} \lesssim 134 \mathrm{GeV}$ ).

For all systems, the overall loss of Higgs signal events due to the acceptance and kinematical cuts (i.e., without accounting for (mis)identification efficiencies) is around a factor of 2 , whereas the backgrounds are reduced by factors of $30-100$, resulting in a final $\mathcal{S} / \mathcal{B} \approx 1$ for all colliding species.

The current analysis is based on LO estimates for the signal and background cross sections. The contributions from higher-order corrections to $\gamma \gamma \rightarrow(\mathrm{H}) \rightarrow \mathrm{b} \bar{b}$ and $\gamma \gamma \rightarrow b \bar{b}$, which can be significant in some regions of phase space, have been studied in detail in [14] for the photon linear collider case. Our applied selection criteria, in particular the exclusive back-to-back two-jets requirement and the $|\cos \theta|<0.5$ cut, effectively remove most of such higher-order contributions. Also, we note that any theoretical uncertainty on the photon fluxes impacts in a similar way the expected yields for signal and background and thereby leaves the $\mathcal{S} / \mathcal{B}$ ratio basically unaffected. Last but not least, more advanced multivariate studies could be contemplated, rather than the simpler "cut-based" criteria applied here, that could further improve the separation of signal over background.

Table II lists the cross sections after each event selection step, as well as the final number of events expected (for the nominal integrated luminosities per run) for signal and backgrounds in the systems with larger signal strength at each collider (Fig. 2, right): Ar-Ar at $\sqrt{s_{\mathrm{NN}}}=6.3 \mathrm{TeV}, \mathrm{Kr}-$ $\mathrm{Kr}$ at $\sqrt{s_{\mathrm{NN}}}=12.5 \mathrm{TeV}$, and $\mathrm{Pb}-\mathrm{Pb}$ at $\sqrt{s_{\mathrm{NN}}}=39 \mathrm{TeV}$, as well as the full $\mathrm{MC}$ results obtained for $\mathrm{Pb}-\mathrm{Pb}$ and $\mathrm{p}-\mathrm{Pb}$ at $\sqrt{s_{\mathrm{NN}}}=39,63 \mathrm{TeV}$ first discussed in [12]. The last column of Table II lists the final number of signal and background events expected after all selection criteria for the nominal 1 -month $\left(10^{6} \mathrm{~s}\right)$ run operation. The expected number of Higgs per month, after cuts, at the HL-LHC and HE-LHC are below unity, whereas one expects 5-20 reconstructed $\mathrm{H}(\mathrm{b} \bar{b})$ events at the FCC.

TABLE II. Summary of the cross sections after each event selection step (see text for details) and final number of events expected (for the nominal integrated luminosities quoted) for signal $\left(N_{\text {Higgs }}\right)$ and backgrounds $\left(N_{\text {backg }}\right)$ in the $\gamma \gamma \rightarrow \mathrm{H}(\mathrm{b} \bar{b})$ measurements in $\mathrm{Ar}-\mathrm{Ar}$ at $\mathrm{HL}-\mathrm{LHC}, \mathrm{Kr}-\mathrm{Kr}$ at $\mathrm{HE}-\mathrm{LHC}$, and $\mathrm{Pb}-\mathrm{Pb}$ and $\mathrm{p}-\mathrm{Pb}$ at FCC.

\begin{tabular}{|c|c|c|c|}
\hline Ar-Ar at $\sqrt{s_{\mathrm{NN}}}=6.3 \mathrm{TeV}$ & $\begin{array}{c}\text { Cross section } \\
\text { (b-jet (mis)tag effic.) }\end{array}$ & $\begin{array}{l}\text { Visible cross section } \\
\text { after } \eta^{j}, p_{T}^{j},|\cos \theta|, m_{j j} \text { cuts }\end{array}$ & $\begin{array}{c}N_{\text {Higgs }}, N_{\text {backg }} \\
\left(\mathcal{L}_{\text {int }}=1.1 \mathrm{pb}^{-1}\right)\end{array}$ \\
\hline$\gamma \gamma \rightarrow \mathrm{H}_{-} \rightarrow \mathrm{b} \overline{\mathrm{b}}$ & $0.20 \mathrm{pb}(0.10 \mathrm{pb})$ & $0.045 \mathrm{pb}$ & 0.05 \\
\hline$\gamma \gamma \rightarrow \mathrm{b} \overline{\mathrm{b}}\left[\mathrm{m}_{\mathrm{b} \overline{\mathrm{b}}}=100-150 \mathrm{GeV}\right]$ & $8.2 \mathrm{pb}(4.0 \mathrm{pb})$ & $0.06 \mathrm{pb}$ & 0.06 \\
\hline$\gamma \gamma \rightarrow \mathrm{c} \overline{\mathrm{c}}\left[\mathrm{m}_{\mathrm{c} \overline{\mathrm{c}}}=100-150 \mathrm{GeV}\right]$ & $61 \mathrm{pb}(0.15 \mathrm{pb})$ & $0.006 \mathrm{pb}$ & 0.006 \\
\hline$\gamma \gamma \rightarrow \mathrm{q} \overline{\mathrm{q}}\left[\mathrm{m}_{\mathrm{q} \overline{\mathrm{q}}}=100-150 \mathrm{GeV}\right]$ & $70 \mathrm{pb}(0.016 \mathrm{pb})$ & $<10^{-3}$ & $<10^{-3}$ \\
\hline $\mathrm{Kr}-\mathrm{Kr}$ at $\sqrt{s_{\mathrm{NN}}}=12.5 \mathrm{TeV}$ & & & $\begin{array}{c}N_{\text {Higgs }} N_{\text {backg }} \\
\left(\mathcal{L}_{\text {int }}=0.12 \mathrm{pb}^{-1}\right)\end{array}$ \\
\hline$\gamma \gamma \rightarrow \mathrm{H}_{-} \rightarrow \mathrm{b} \overline{\mathrm{b}}$ & $11 \mathrm{pb}(5.5 \mathrm{pb})$ & $2.5 \mathrm{pb}$ & 0.30 \\
\hline$\gamma \gamma \rightarrow \mathrm{b} \overline{\mathrm{b}}\left[\mathrm{m}_{\mathrm{b} \overline{\mathrm{b}}}=100-150 \mathrm{GeV}\right]$ & $365 \mathrm{pb}(178 \mathrm{pb})$ & $2.8 \mathrm{pb}$ & 0.34 \\
\hline$\gamma \gamma \rightarrow \mathrm{c} \overline{\mathrm{c}}\left[\mathrm{m}_{\mathrm{c} \overline{\mathrm{c}}}=100-150 \mathrm{GeV}\right]$ & $2.7 \mathrm{nb}(6.7 \mathrm{pb})$ & $0.24 \mathrm{pb}$ & 0.03 \\
\hline$\gamma \gamma \rightarrow \mathrm{q} \overline{\mathrm{q}}\left[\mathrm{m}_{\mathrm{q} \overline{\mathrm{q}}}=100-150 \mathrm{GeV}\right]$ & $3.1 \mathrm{nb}(0.70 \mathrm{pb})$ & $<10^{-3}$ & $<10^{-4}$ \\
\hline $\mathrm{Pb}-\mathrm{Pb}$ at $\sqrt{s_{\mathrm{NN}}}=39 \mathrm{TeV}$ & & & $\begin{array}{c}N_{\text {Higgs }} N_{\text {backg }} \\
\left(\mathcal{L}_{\text {int }}=110 \mathrm{nb}^{-1}\right)\end{array}$ \\
\hline$\gamma \gamma \rightarrow \mathrm{H} \rightarrow \mathrm{b} \overline{\mathrm{b}}$ & $1.0 \mathrm{nb}(0.50 \mathrm{nb})$ & $0.19 \mathrm{nb}$ & 21.1 \\
\hline$\gamma \gamma \rightarrow \mathrm{b} \overline{\mathrm{b}}\left[\mathrm{m}_{\mathrm{b} \overline{\mathrm{b}}}=100-150 \mathrm{GeV}\right]$ & $24.3 \mathrm{nb}(11.9 \mathrm{nb})$ & $0.23 \mathrm{nb}$ & 25.7 \\
\hline$\gamma \gamma \rightarrow \mathrm{c} \overline{\mathrm{c}}\left[\mathrm{m}_{\mathrm{c} \overline{\mathrm{c}}}=100-150 \mathrm{GeV}\right]$ & $525 \mathrm{nb}(1.31 \mathrm{nb})$ & $0.02 \mathrm{nb}$ & 2.3 \\
\hline$\gamma \gamma \rightarrow \mathrm{q} \overline{\mathrm{q}}\left[\mathrm{m}_{\mathrm{q} \overline{\mathrm{q}}}=100-150 \mathrm{GeV}\right]$ & $590 \mathrm{nb}(0.13 \mathrm{nb})$ & $0.002 \mathrm{nb}$ & 0.25 \\
\hline $\mathrm{p}-\mathrm{Pb}$ at $\sqrt{s_{\mathrm{NN}}}=63 \mathrm{TeV}$ & & & $\begin{array}{l}N_{\text {Higgs }} N_{\text {backg }} \\
\left(\mathcal{L}_{\text {int }}=29 \mathrm{pb}^{-1}\right)\end{array}$ \\
\hline$\gamma \gamma \rightarrow \mathrm{H}_{-} \rightarrow \mathrm{b} \overline{\mathrm{b}}$ & $0.87 \mathrm{pb}(0.42 \mathrm{pb})$ & $0.16 \mathrm{pb}$ & 4.8 \\
\hline$\gamma \gamma \rightarrow \mathrm{b} \overline{\mathrm{b}}\left[\mathrm{m}_{\mathrm{b} \overline{\mathrm{b}}}=100-150 \mathrm{GeV}\right]$ & $21.8 \mathrm{pb}(10.7 \mathrm{pb})$ & $0.22 \mathrm{pb}$ & 6.3 \\
\hline$\gamma \gamma \rightarrow \mathrm{c} \overline{\mathrm{c}}\left[\mathrm{m}_{\mathrm{c} \overline{\mathrm{c}}}=100-150 \mathrm{GeV}\right]$ & $410 \mathrm{pb}(1.03 \mathrm{pb})$ & $0.011 \mathrm{pb}$ & 0.3 \\
\hline$\gamma \gamma \rightarrow \mathrm{q} \overline{\mathrm{q}}\left[\mathrm{m}_{\mathrm{q} \overline{\mathrm{q}}}=100-150 \mathrm{GeV}\right]$ & $510 \mathrm{pb}(0.114 \mathrm{pb})$ & $0.001 \mathrm{pb}$ & 0.04 \\
\hline
\end{tabular}



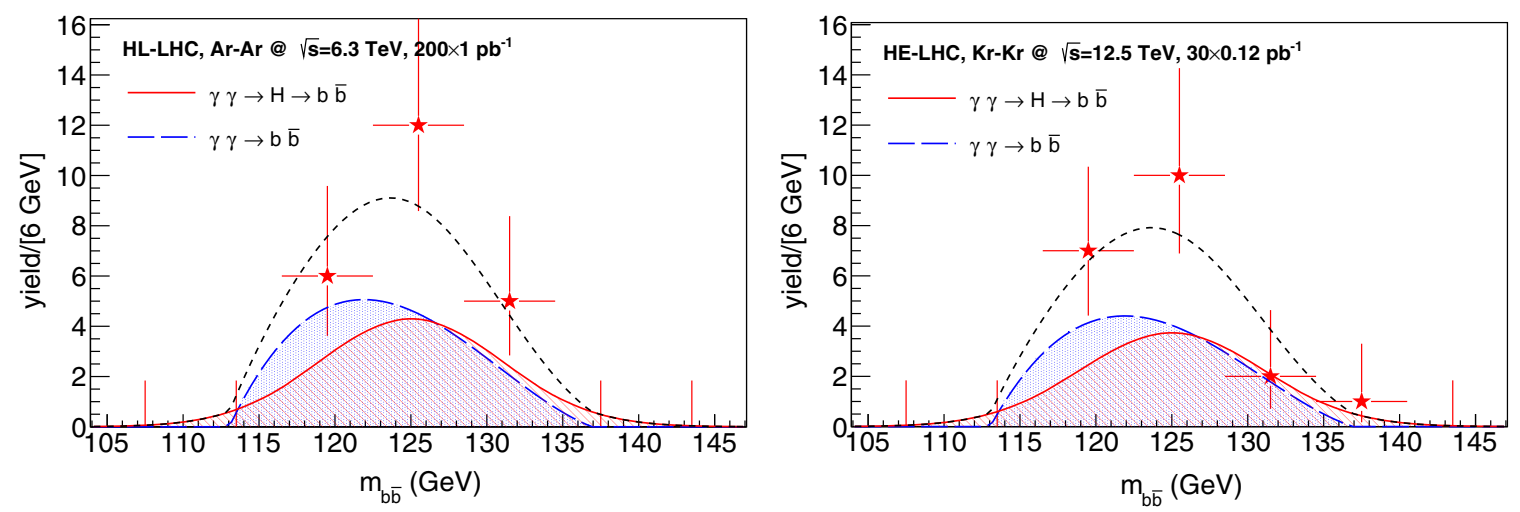

FIG. 3. Expected invariant mass distributions for b-jet pairs from the photon-fusion Higgs signal (hatched red Gaussian) and b $\bar{b}+$ $\mathrm{c} \overline{\mathrm{c}}+\mathrm{q} \overline{\mathrm{q}}$ continuum (hatched blue area) in ultraperipheral $\operatorname{Ar}-\mathrm{Ar}\left(\sqrt{s_{\mathrm{NN}}}=6.3 \mathrm{TeV}\right.$, left $)$ and $\mathrm{Kr}-\mathrm{Kr}\left(\sqrt{s_{\mathrm{NN}}}=12.5 \mathrm{TeV}\right.$, right $)$ collisions, after event selection criteria and with the quoted integrated luminosities (see text). The red stars show the expected signalplus-background invariant mass counts. The dashed black curve corresponds to the sum of theoretical signal and background yields.

The final significance of the signal can be derived from the number of counts within $\pm 1.4 \sigma_{j j}$ around the Gaussian Higgs peak (i.e., $116 \lesssim m_{\mathrm{b} \overline{\mathrm{b}}} \lesssim 134 \mathrm{GeV}$ ) over the dijet continuum remaining after cuts. In a simplified cut-andcount approach, one can estimate the statistical sample increase needed for a $3 \sigma$ evidence from the $N_{\text {Higgs }}$ values listed in the last column of Table II. For an integrated luminosity 200 times larger than the nominal for $\mathrm{Ar}-\mathrm{Ar}$ (6.3 TeV), one has $\mathcal{S} / \sqrt{\mathcal{B}} \approx 10 / \sqrt{12} \approx 3$. The same numbers at the HE-LHC for 30 times more luminosity integrated in $\operatorname{Kr}-\operatorname{Kr}(12.5 \mathrm{TeV})$, yield $\mathcal{S} / \sqrt{\mathcal{B}} \approx 9 / \sqrt{10} \approx 3$. Thus, reaching $3 \sigma$ evidence of UPC Higgs-production at HL-LHC and at HE-LHC requires at least factors of $\times 200$ and $\times 30$ more integrated luminosities in $\mathrm{Ar}-\mathrm{Ar}$ and $\mathrm{Kr}-\mathrm{Kr}$ collisions, respectively, than currently designed. Figure 3 shows the expected invariant dijet mass distributions after selection criteria for signal and backgrounds at the HLLHC (Ar-Ar, left) and HE-LHC (Kr-Kr, right) for such increased integrated luminosities. A factor of 10 increase in $\mathcal{L}_{\text {int }}$ could be gained at both colliders simply by running during the time $\left(10^{7} \mathrm{~s}\right)$ typical of a proton-proton run, instead of the nominal 1-month $\left(10^{6} \mathrm{~s}\right)$ heavy-ion run operation. Such a longer run, motivated by Higgs- rather than heavy-ion physics, would allow for an evidence of the process at HE-LHC, by combining three experiments (or over three runs in a single one). Achieving the same significance at the HL-LHC seems out of reach unless an extra factor of $\times 20$ in the instantaneous Ar-Ar luminosity is accomplished by some currently unidentified means. In any case, going from the simple evidence to a
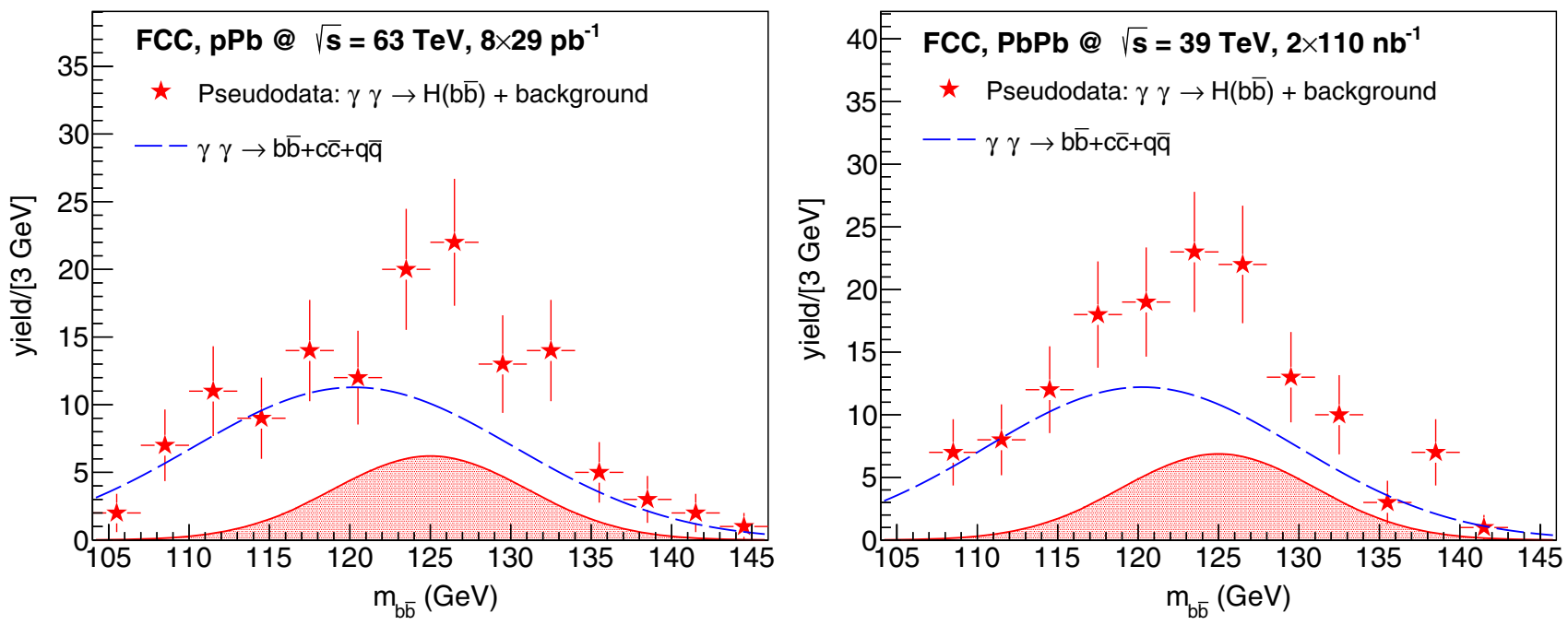

FIG. 4. Expected invariant mass distributions for b-jet pairs from the photon-fusion Higgs signal (hatched red Gaussian) and $b \bar{b}+$ $\mathrm{c} \overline{\mathrm{c}}+\mathrm{q} \overline{\mathrm{q}}$ continuum (blue curve) in ultraperipheral $\mathrm{p}-\mathrm{Pb}\left(\sqrt{s_{\mathrm{NN}}}=63 \mathrm{TeV}\right.$, left $)$ and $\mathrm{Pb}-\mathrm{Pb}\left(\sqrt{s_{\mathrm{NN}}}=39 \mathrm{TeV}\right.$, right $)$ collisions after event selection criteria and with the quoted integrated luminosities (see text). The red stars show the expected signal-plus-background invariant mass counts. 
$5 \sigma$ observation would require yet another extra $(5 / 3)^{2} \approx$ 2.8 increase in the collected data. These estimates indicate that the UPC Higgs observation will very likely remain elusive at the HL-LHC and HE-LHC.

The situation appears much more favorable at the FCC thanks to the factors of 10 and 100 larger Higgs cross sections, and factors of 10 increased instantaneous luminosities, compared to the HE-LHC and HL-LHC. Figure 4 presents the expected double $\mathrm{b}$-jet invariant mass distributions in $\mathrm{p}-\mathrm{Pb}$ (left) and $\mathrm{Pb}-\mathrm{Pb}$ (right) at the FCC. Lead-lead collisions at $\sqrt{s}=39 \mathrm{GeV}$ with the nominal integrated luminosity of $\mathcal{L}_{\text {int }}=110 \mathrm{nb}^{-1}$ per month yield $\sim 20$ signal counts over about the same number for the sum of backgrounds in a $m_{\mathrm{b} \overline{\mathrm{b}}} \approx$ $116-134 \mathrm{GeV}$ window. Reaching a $5 \sigma$ statistical significance would just require to combine the measurements of the first run from two different experiments or accumulating two 1-month runs in a single one (Fig. 4, right). Similar estimates for $\mathrm{p}-\mathrm{Pb}$ at $63 \mathrm{TeV}$ yield about five signal events after cuts over a background of seven continuum events for the design $\mathcal{L}_{\text {int }}=29 \mathrm{pb}^{-1}$. Reaching a $5 \sigma$ observation of $\gamma \gamma \rightarrow \mathrm{H}$ production requires in this case to run for $\sim 8$ months $\left(10^{7} \mathrm{~s}\right)$, instead of the nominal 1 month, or running 4 months and combining two experiments (Fig. 4, left).

All the derived number of events and significances are based on the aforementioned simple set of kinematical cuts and signal-over-background estimates and can be likely further improved by using more advanced multivariate studies and a full parametric shape analysis for the significance calculation. Notwithstanding such potential improvements, the numbers presented here provide realistic estimates of the feasibility of the UPC Higgs boson measurements at all currently (planned or under consideration) future CERN hadron colliders.

\section{SUMMARY}

We have presented prospect studies for the measurement of the two-photon production of the Higgs boson in ultraperipheral $\mathrm{Pb}-\mathrm{Pb}$, $\mathrm{Xe}-\mathrm{Xe}, \mathrm{Kr}-\mathrm{Kr}$, Ar-Ar, O-O, p-Pb, and p-p collisions at three planned CERN future hadron colliders: HL-LHC, HE-LHC, and FCC. Cross sections have been obtained with MADGRAPH 5, modified to include the corresponding nuclear equivalent photon fluxes with no hadronic overlap of the colliding beams, for nucleon-nucleon c.m. energies over $\sqrt{s_{\mathrm{NN}}}=3-100 \mathrm{TeV}$. The Higgs cross sections roughly rise by a factor of 10 (100) when increasing the c.m. energy from the HL-LHC to the HE-LHC (FCC). At the HL$\mathrm{LHC}$ and HE-LHC, although $\mathrm{Pb}-\mathrm{Pb}$ features the largest Higgs cross section, $\sigma(\gamma \gamma \rightarrow \mathrm{H})=15$, and $150 \mathrm{pb}$ thanks to its $Z^{4}$-amplified photon fluxes, the most competitive systems to try a measurement of UPC Higgs production are Ar-Ar and $\mathrm{Kr}-\mathrm{Kr}$, respectively, thanks to the larger available beam luminosities for such lighter species.

The observation of the Higgs boson in UPCs, via its dominant $b \bar{b}$ decay channel, relies on the measurement of two exclusive b-jets with invariant masses peaked at $m_{\mathrm{H}}$, on top of a background of $\gamma \gamma \rightarrow b \bar{b}, c \bar{c}, q \bar{q}$ continuum pairs, where charm and light $(\mathrm{q}=\mathrm{u}, \mathrm{d}, \mathrm{s})$ quarks are misidentified as b-quarks. The same MADGRAPH 5 setup used to compute Higgs cross sections and generate the corresponding events has been employed for the exclusive two-photon production of $b \bar{b}$, c $\bar{c}$, and $q \bar{q}$ dijets. The HL-LHC and HE-LHC analyses have been carried out at the parton level, whereas for FCC energies the b-quarks have been showered and hadronized with PYTHIA 8, and reconstructed in a exclusive two-jet final-state with the $k_{T}$ algorithm. Given the simplicity of the exclusive final states considered, no significant differences between hadron- and parton-level results exist. By assuming realistic jet acceptance, reconstruction performances, and (mis)tagging efficiencies, and applying appropriate kinematical cuts on the jet $p_{T}$ and angles, it has been shown that the $\mathrm{H}(\mathrm{b} \overline{\mathrm{b}})$ signal can be reconstructed on top of the $\gamma \gamma \rightarrow b \bar{b}, c \bar{c}, q \bar{q}$ continuum backgrounds. On the one hand, reaching $3 \sigma$ evidence of UPC Higgs-production at HL-LHC and at HE-LHC requires factors of about $\times 200$ and $\times 30$ more integrated luminosities in $\mathrm{Ar}-\mathrm{Ar}$ and $\mathrm{Kr}-\mathrm{Kr}$ collisions, respectively, than currently planned for both machines. Factors of 10 in integrated luminosity can be gained running for the duration $\left(10^{7} \mathrm{~s}\right)$ typical of a protonproton run, rather than the nominal 1-month heavy-ion operation. This would open up the possibility of a $3 \sigma$ evidence at the HE-LHC, but would still fall too short for any feasible measurement at the HL-LHC. On the other hand, the measurement of $\gamma \gamma \rightarrow \mathrm{H} \rightarrow \mathrm{b} \overline{\mathrm{b}}$ would yield about 20 (5) signal counts after cuts in $\mathrm{Pb}-\mathrm{Pb}$ (p-Pb) collisions for their nominal integrated luminosities per run. Observation of the photon-fusion Higgs production at the $5 \sigma$ level is achievable in the first FCC run by combining the measurements of two experiments (or doubling the luminosity in a single one) in $\mathrm{Pb}-\mathrm{Pb}$ and by running for about 8 months (or 4 months and combining two experiments) in the $\mathrm{p}-\mathrm{Pb}$ case. The feasibility studies presented here indicate the Higgs physics potential open to study in $\gamma \gamma$ ultraperipheral ion collisions at current and future CERN hadron colliders, eventually providing an independent measurement of the $\mathrm{H}-\gamma$ coupling not based on Higgs decays but on a $s$-channel production mode, as well as of its total width combining the photon-fusion measurement with the $\mathrm{H} \rightarrow \gamma \gamma$ decay branching ratio accessible at a future $e^{+} e^{-}$collider.

\section{ACKNOWLEDGMENTS}

P. R. T. acknowledges financial support from CERN (TH Department and FCC project) as well as from the PAPD/ FAPERJ program (Brazil). D. E. Martins acknowledges financial support from the CAPES/INCT-FNA (number 88887.461636/2019-00) program (Brazil). We thank I. Helenius and L. Harland-Lang for useful discussions on PYTHIA8 and/or photon-photon collisions, as well as J. Jowett for feedback on running conditions for light-ions at the HL-LHC and HE-LHC, and E. Chapon on statistical methods. 
[1] C. von Weizsäcker, Z. Physik 88, 612 (1934); E. J. Williams, Phys. Rev. 45, 729 (1934); E. Fermi, Nuovo Cimento 2, 143 (1925).

[2] C. A. Bertulani and G. Baur, Phys. Rep. 163, 299 (1988).

[3] A. J. Baltz et al., Phys. Rep. 458, 1 (2008).

[4] Z. Citron et al., CERN Yellow Rep. Monogr. 7, 1159 (2019).

[5] A. Abada et al. (FCC Collaboration), Eur. Phys. J. ST 228, 1109 (2019).

[6] A. Dainese et al., CERN Yellow Rep. 3, 635 (2017); D. d'Enterria et al., Nucl. Phys. A967, 888 (2017); A. Abada et al. (FCC Collaboration), Eur. Phys. J. ST 228, 755 (2019).

[7] M. G. Albrow et al. (FP420 R\&D Collaboration), J. Instrum. 4, T10001 (2009).

[8] S. Chatrchyan et al. (CMS Collaboration), Phys. Lett. B 716, 30 (2012); G. Aad et al. (ATLAS Collaboration), Phys. Lett. B 716, 1 (2012).

[9] D. L. Borden, D. A. Bauer, and D. O. Caldwell, Phys. Rev. D 48, 4018 (1993).

[10] M. Grabiak, B. Muller, W. Greiner, G. Soff, and P. Koch, J. Phys. G 15, L25 (1989); E. Papageorgiu, Phys. Rev. D 40, 92 (1989); M. Drees, J. Ellis, and D. Zeppenfeld, Phys. Lett. B 223, 454 (1989); K. J. Abraham, R. Laterveer, J. A. M. Vermaseren, and D. Zeppenfeld, Phys. Lett. B 251, 186 (1990).

[11] D. d'Enterria and J. P. Lansberg, Phys. Rev. D 81, 014004 (2010).

[12] D. d'Enterria, D. E. Martins, and P. Rebello Teles, CERN Proc. 1, 33 (2018).

[13] J. F. Gunion and H. E. Haber, Phys. Rev. D 48, 5109 (1993); E. Boos et al., Nucl. Instrum. Methods A 472, 100 (2001).

[14] M. Melles and W. J. Stirling, Phys. Rev. D 59, 094009 (1999); B. Kamal, Z. Merebashvili, and A. P. Contogouris, Phys. Rev. D 51, 4808 (1995); 55, 3229(E) (1997); G. Jikia and A. Tkabladze, Phys. Rev. D 54, 2030 (1996).
[15] J. Alwall, R. Frederix, S. Frixione, V. Hirschi, F. Maltoni, O. Mattelaer, H.-S. Shao, T. Stelzer, P. Torrielli, and M. Zaro, J. High Energy Phys. 07 (2014) 079.

[16] M. A. Shifman, A. I. Vainshtein, M. B. Voloshin, and V. I. Zakharov, Yad. Fiz. 30, 1368 (1979) [Sov. J. Nucl. Phys. 30, 711 (1979)]; B. A. Kniehl and M. Spira, Z. Phys. C 69, 77 (1995); S. Dawson and R. Kauffman, Phys. Rev. D 49, 2298 (1994).

[17] V. M. Budnev, I. F. Ginzburg, G. V. Meledin, and V. G. Serbo, Phys. Rep. 15, 181 (1975).

[18] J. D. Jackson, Classical Electrodynamics, 2nd ed. (John Wiley \& Sons, New York, 1975).

[19] H. De Vries, C. W. De Jager, and C. De Vries, At. Data Nucl. Data Tables 36, 495 (1987).

[20] R. N. Cahn and J. D. Jackson, Phys. Rev. D 42, 3690 (1990).

[21] M. Spira, Nucl. Instrum. Methods A 389, 357 (1997); A. Djouadi, J. Kalinowski, and M. Spira, Comput. Phys. Commun. 108, 56 (1998); A. Djouadi, J. Kalinowski, M. Mühlleitner, and M. Spira, arXiv:1003.1643.

[22] T. Sjöstrand, S. Ask, J. R. Christiansen, R. Corke, N. Desai, P. Ilten, S. Mrenna, S. Prestel, C. O. Rasmussen, and P. Z. Skands, Comput. Phys. Commun. 191, 159 (2015).

[23] S. Catani, Y. L. Dokshitzer, M. H. Seymour, and B. R. Webber, Nucl. Phys. B406, 187 (1993).

[24] M. Cacciari, G. P. Salam, and G. Soyez, Eur. Phys. J. C 72, 1896 (2012).

[25] P. Rebello Teles and D. d'Enterria, arXiv:1510.08141.

[26] D. d'Enterria, P. Rebello Teles, and D. E. Martins, arXiv: 1712.07023.

[27] S. R. Klein, J. Nystrand, J. Seger, Y. Gorbunov, and J. Butterworth, Comput. Phys. Commun. 212, 258 (2017).

[28] L. A. Harland-Lang, V. A. Khoze, and M. G. Ryskin, Eur. Phys. J. C 79, 39 (2019).

[29] A. M. Sirunyan et al. (CMS Collaboration), J. Instrum. 13, P05011 (2018). 